\title{
GLOBAL EFFECTS OF INTERACTIONS ON GALAXY EVOLUTION
}

\author{
Robert C. Kennicutt, Jr. \\ Steward Observatory, University of Arizona \\ Tucson, Arizona, USA
}

\begin{abstract}
Recent observations of the evolutionary properties of paired and interacting galaxies are reviewed, with special emphasis on their global emission properties and star formation rates. Data at several wavelengths provide strong confirmation of the hypothesis, proposed originally by Larson and Tinsley, that interactions trigger global bursts of star formation in galaxies. The nature and properties of the starbursts, and their overall role in galactic evolution will also be discussed.
\end{abstract}

\section{INTRODUCTION}

The preponderance of tidally disturbed pairs of galaxies among samples of starbursting objects such as Markarian galaxies (Karachentsev and Karachentseva 1974), emissionline galaxies (Wasilewski 1983), and the ultraluminous IRAS galaxies (Soifer et al. 1984) shows that interactions can be a significant, if not dominant factor in galaxy evolution. The quantitative foundation for our understanding of the relationship between interactions and global star formation in galaxies was laid out in an influential paper by Larson and Tinsley (1978). "LT" demonstrated that interacting galaxies possess an unusually large dispersion in UBV colors, which they attributed to global bursts of star formation, and by fitting simple color evolution models to these observations they were able to derive the first quantitative estimates of the amplitudes and lifetimes of the starbursts. In retrospect it is fair to say that this paper had as stimulating an effect on the observers' community that the famous Toomre and Toomre (1972) paper had for theoretical work in the field.

The main goal of this review is to summarize what we have learned since 1978 about the evolution of interacting galaxies, with special emphasis on their global star formation properties, an area where much progress has been made. Several surveys of the integrated emission properties of interacting galaxies at visible, infrared, and radio wavelengths are now available, and these provide a self-consistent picture of how interactions affect the stellar birthrate in galaxies. I will also briefly discuss what can be learned about the starbursts from modelling the observations; these analyses not only provide us with information on the temporal properties of the bursts, but they also allow us to address such fundamental questions as whether the star formation in the bursts differs differs in any fundamental way (e.g., efficiency, IMF) from the steady state star formation in galaxies, or whether interactions trigger a significant fraction of the star formation in the universe, either now or at earlier cosmological epochs.

Throughout this paper I will emphasize the global effects of interactions on galaxy evolution; a companion review by Heckman discusses the effects of interactions on nuclear activity and star formation. Other topics which I will only address briefly, since they are reviewed elsewhere, include the morphologies, kinematics, and gas distributions in interacting galaxies (see papers by Karachentsev and Hickson), and the role of interactions and mergers in the formation of elliptical galaxies and spiral bulges. The latter subject deserves a review of its own, and for that $I$ refer the reader to a lively and provocative paper by Schweizer (1990). 


\section{INTEGRATED PROPERTIES OF PAIRED AND INTERACTING GALAXIES}

Recent observations provide a compelling case for a link between close tidal interactions and large star formation bursts. Since the original LT analysis two key improvements have been made, the development of optical, infrared, and radio tracers of the young star forming populations, and the observation of complete samples of paired and isolated galaxies, which allows us to derive unbiased statistics on the global role of interactions for galactic evolution.

\section{UBV Colors and Surface Photometry}

Since the LT study integrated multicolor photometry has been obtained for a considerably larger number of interacting galaxies. Observations of northern galaxies have been compiled by Demin, Dibai, and Tomov (1981) and Arkhipova (1982). The Demin et al. paper includes a recalibration of the previously published photometry of Tomov, and supercedes those earlier studies. A longstanding program of photometry of southern pairs has been conducted by the ESO/Uppsala group, as part of their large southern galaxy survey (e.g., Ardeberg and Bergvall 1977, Bergvall et al. 1978, Johansson 1988). These data confirm the large color dispersion which characterizes close pairs, and the extremely blue colors of the most active starbursting systems.

The advent of wide-field CCD cameras has recently led to a considerable body of data on the luminosity and color distributions of interacting systems (e.g., Armus, Heckman, and Miley 1987, Friedman et al. 1987, Fried and Lutz 1988, Bothun et al. 1989, Schombert, Wallin, and Struck-Marcell 1990). The Schombert et al. study is of particular interest, because it provides the first comprehensive data on the photometric properties of the faint tidal bridges, tails, and envelopes in close pairs. A focal reducer camera on the Palomar $1.5 \mathrm{~m}$ telescope was used to obtain deep BVri surface photometry to study the structure and colors of the tidal features in 25 pairs selected from the Arp (1966) atlas. Several interesting new results emerge from this study. The tidal features contain a large fraction of the total luminosity of the systems, $25 \%$ on average, and up to $60 \%$ in extreme cases. The tidal bridges, tails, and plumes are systematically bluer than the parent galaxies, with colors that indicate that excess star formation, rather than low metallicity, is responsible for most of the difference. This would suggest that active star formation has persisted over very long $\left(\sim 10^{8} \mathrm{yr}\right)$ timescales in many of the pairs. The Schombert et al. analysis illustrates the rich variety of problems in this area which can now be attacked with CCD imagers on modest aperture telescopes, especially when the data are combined with color evolution models and kinematic observations.

\section{$\underline{\mathrm{H} \alpha}$ Emission and Massive Star Formation Rates}

The colors of interacting galaxies provide strong evidence for abnormal star formation rates (SFRs), but the quantitative interpretation of such data is limited. Integrated colors are only weakly dependent on the current SFR (e.g., Searle, Sargent, and Bagnuolo 1973), and they are subject to large systematic errors due to variations in reddening and metallicity. On the other hand the luminosity of a hydrogen recombination line such as $\mathrm{H} \alpha$ provides a direct, quantitative tracer of the SFR for massive stars $\left(>10 M_{\odot}\right)$, and when combined with the UBV data can provide quantitative estimates of the SFR integrated over all stellar masses (Kennicutt 1983, Gallagher et al. 1984).

$\mathrm{H} \alpha$ surveys of paired and interacting galaxies have been made by Dostal' (1982), Bushouse (1987) and Kennicutt et al. (1987). Although the techniques used in these surveys were similar, the sample selection criteria were quite different. Dostal' and Bushouse studied unusually perturbed pairs, using the morphological descriptions in the V-V and UGC catalogs for the initial selection. This provides an uncontaminated sample of pairs which probe the maximum effects of interactions on the SFR. Kennicutt et al. observed a similar sample of disturbed systems drawn from the Arp atlas, but they also formed a complete magnitude-limited sample of close spiral and irregular pairs, using a statistically generated 
catalog of van Albada (private communication), combined with redshift information to eliminate visual pairs. The complete sample provides a reliable assessment of the overall influence of interactions on paired galaxies.

All of these samples show evidence of abnormally high SFRs. As an example, Figure

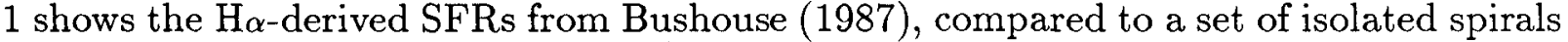
taken from Kennicutt and Kent (1983). The median $\mathrm{H} \alpha$ fluxes, normalized to the disk area or to the red continuum luminosity of the galaxies, show enhancements ranging from $30 \%$ in the complete pairs sample of Kennicutt et al. to factors of 2.2-2.5 for the morphologically selected samples. Note that the use of normalized luminosities minimizes any Malmquist bias or other observational selection effects in the comparisons.
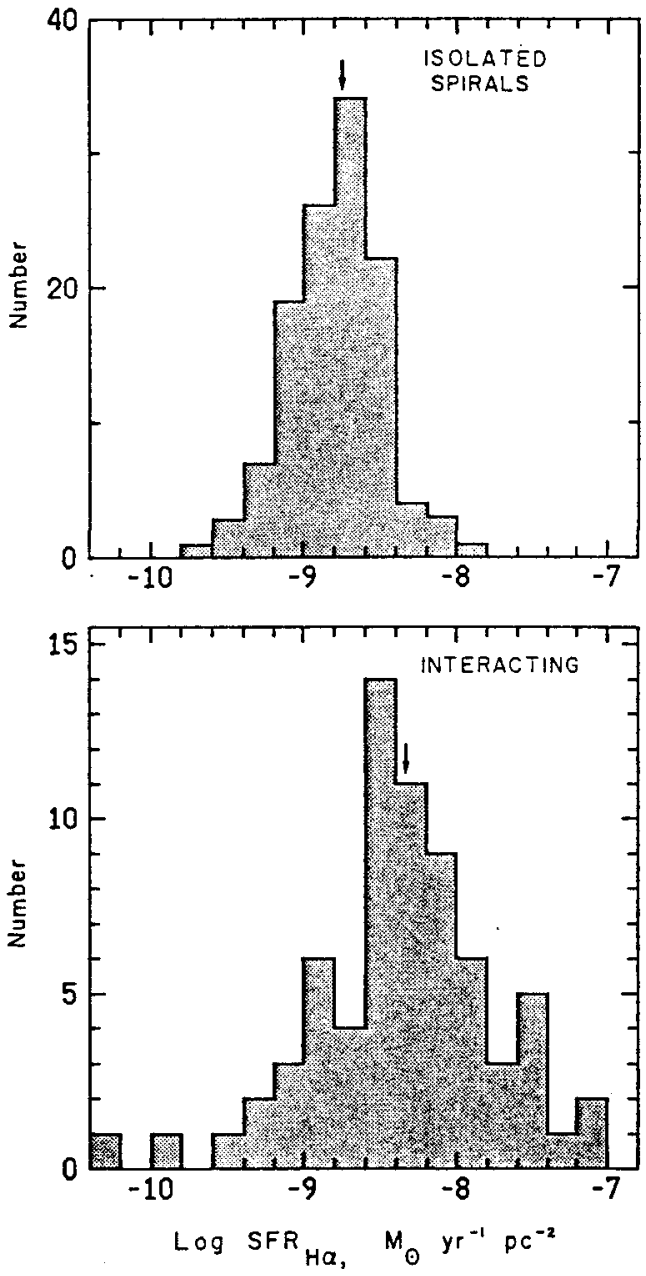

Fig. 1. Comparison of SFRs (normalized to disk area) for a sample of highly perturbed interacting galaxies, and a comparison sample of isolated galaxies, from Bushouse (1987).

It is evident from Figure 1 that the response of individual galaxies varies enormously. Several factors contribute to this dispersion, including the wide range of morphological types and gas content, the range of evolutionary stages (relative to the peak star formation episode), and a real variation in peak response to the interactions (see Sec. IV). Of special interest are the most active, starbursting systems. Inspection of Figure 1 reveals that there is a subpopulation of extreme emission-line galaxies which is almost uniquely associated with interactions. For example, galaxies with $\mathrm{H} \alpha$ equivalent widths above $50 \AA$ comprise a quarter of the Kennicutt et al. (1987) sample, as opposed to only $4 \%$ among isolated galaxies. This is far more than can be accounted for by a mere shifting of the emission distribution of normal 
galaxies to a higher median level, and it suggests a highly nonlinear response of the SFR to the dynamical stimulation in many systems. On the other hand, it is important to remember that such starbursting systems are extremely rare; the average SFR enhancement in these samples, even in the most disturbed systems, is never much more than a factor of two.

Most of the $\mathrm{H} \alpha$ data were obtained with imaging detectors, so they also provide information on the spatial distribution and luminosity functions of the star forming regions. Perhaps the most interesting result is a strong tendency for the star formation in interacting spirals to be concentrated toward the nucleus (Bushouse 1987, Kennicutt et al. 1987). There is also a significant correlation between the level of activity in the nuclei and disks, above what is normally observed along the Hubble sequence (see Heckman's review for more details). Less is known about the azimuthal variation of the SFR in interacting galaxies. Hodge (1975) analyzed the H II region distributions in 14 systems and found severe distortions in several. In the Kennicutt et al. sample there is a qualitative trend of unusually strong spiral patterns in the close pairs, consistent with tidal triggering or 'swing amplification' of spiral density waves.

The trends observed in the total SFRs of interacting galaxies are also manifested in the properties of their largest H II region complexes. Petrosian, Saakian, and Khachikian (1985) have shown that the frequency of giant H II complexes or "superassociations" is twice as high in close pairs as in isolated galaxies. A detailed follow-up to this study is being pursued by Keel and collaborators.

\section{Far-Infrared Properties}

Currently the most active area of interest in this field is the far-infrared (FIR) emission of interacting galaxies, especially the extraordinary IR-luminous interacting/merging systems. Several recent reviews of this subject are available (e.g., Joseph 1987, 1988, Telesco 1988), and I will limit my discussion to the evidence for extranuclear star formation.

As with the $\mathrm{H} \alpha$ studies described above, the FIR surveys can be loosely divided into two groups according to the selection criteria applied, either interacting galaxies chosen by morphological criteria (e.g., Joseph et al. 1984, Lonsdale, Persson, and Mathews 1984; Bushouse, Lamb, and Werner 1988; Telesco, Wolstencroft, and Done 1988; Sulentic 1989), or comparisons of paired and isolated galaxies selected without regard any direct evidence of interactions (e.g., Kennicutt et al. 1987, Haynes and Herter 1988).

Again several common patterns emerge from these studies. The unbiased samples of galaxy pairs show modest (20-60\%) enhancements in average FIR luminosity, and they contain few if any examples of ultraluminous systems. The latter result is consistent with the low space density of the ultraluminous galaxies, and the association of such objects with mergers, rather than discrete pairs. The percentage of excess FIR emission on average is similar the that observed at $\mathrm{H} \alpha$, suggesting that increased star formation is the principle source of the IR excess. The strongly perturbed systems show considerably larger FIR enhancements, by factors of 2-3, and a significant fraction of ultraluminous systems. Only the most strongly interacting systems (or mergers) show extreme values of FIR excess. In parallel with the increase in FIR luminosity, there is a trend toward hotter color temperatures for the emitting dust (e.g., Bushouse et al. 1988, Telesco et al. 1988).

Unfortunately the interpretation of these data is more complicated than for the optical results discussed above. Usually the IRAS beam size at 60 and $100 \mu \mathrm{m}$ is much larger than the galaxies in question (often the entire pair is unresolved), so it is not known a priori whether the excess FIR emission originates in the nuclei or the main bodies of the galaxies. The heating source of the dust is also unclear; massive young stars, the general interstellar radiation field, and nonthermal radiation from the nucleus may all contribute (e.g., Lonsdale and Helou 1987, Walterbos and Schwering 1987). Groundbased 10-20 $\mu \mathrm{m}$ measurements of selected galaxies indicate that a considerable fraction of the FIR emission in the most luminous objects is emitted by the nucleus (e.g., Lonsdale, Persson, and Mathews 1984, Cutri and McAlary 1985). On the other hand high-resolution IR images of systems such 
as Arp 299 (Telesco, Decher, and Gatley 1985) and Arp 118 (Joy and Ghigo 1988) reveal extensive regions of extranuclear emission associated with massive star formation.

Although it is premature to draw firm conclusions from the currently available data, the observations are consistent with enhanced extranuclear SFRs in the interacting galaxies. In contrast to the optical studies, however, which revealed a relatively homogeneous class of star forming (mainly disk) systems, there is evidence from the FIR surveys for a second population of ultraluminous merging galaxies, distinct in terms of not only the FIR luminosity but also their morphologies, kinematics, and spectroscopic properties. The nature and source of the FIR emission in these systems may be quite different from that which characterizes the steady-state emission of normal galaxies.

\section{Radio Continuum Emission and Supernova Rates}

Radio continuum studies of interacting and paired galaxies by Sulentic (1976) and Stocke (1978) provided some the earliest evidence for the abnormal emission properties of these objects. These results have since been confirmed by other workers (e.g., Hummel 1981, Condon et al. 1982). The mean enhancements are similar to those seen at $\mathrm{H} \alpha$ and the FIR, typically a factor of two.

The physical origin of the excess radio emission is less certain. At centimeter wavelengths the overwhelming fraction of the radio emission is nonthermal, and possible causes for the enhanced emission include nuclear emission, magnetic field compression in the disks, not unexpected in strong tidal encounters, or a burst of energetic charged particles from supernovae formed in a starburst. Hummel (1981) obtained spatially resolved maps for complete samples of paired and isolated spirals, and found that most of the excess radio emission in pairs originates in the nuclear regions. Subsequent studies at higher resolution by Condon et al. (1982) and Hummel et al. $(1987,1990)$ confirm the excess nuclear activity in interacting systems, but the question of whether the disk emission is enhanced remains open. A modest excess (30-50\%) in disk emission, consistent with what is seen in $\mathrm{H} \alpha$ and the FIR, is not ruled out by the available data.

Detailed aperture synthesis mapping of nearby systems is beginning to reveal more about the nature of the radio emission. A survey of radio-bright galaxies by Condon (1983) revealed several examples of interacting galaxies with bright radio disks, though Condon has emphasized that an interaction is not a necessary condition for such disks. Hummel and van der Hulst (1986) have published a detailed multi-frequency study of 'The Antennae' (NGC $4038 / 39$ ), and demonstrate that the strongly enhanced nonthermal disk emission in this pair is produced by increases in both the magnetic field strength and the charged particle densities. The latter is consistent with an elevated supernova rate, which in turn is consistent with the blue colors and bright $\mathrm{H} \alpha$ emission in the system. High-resolution radio maps of some nearby starburst and (supposedly) interacting galaxies show direct evidence of high supernova rates. Maps of M82 and NGC 3448 by Kronberg, Biermann, and Schwab (1985) and Noreau and Kronberg (1987) show large numbers of compact, unresolved sources which are almost certainly supernova remnants.

These conclusions are borne out by the statistics of the supernovae themselves in interacting galaxies. Smirnov and Tsvetkov (1981) have compared the supernova rates in isolated, paired, and interacting galaxies, and found that supernovae occur twice as frequently in the interacting systems. These authors are also able to exclude the possibility that the difference in rates arises from observational selection effects.

\section{PECULIAR GALAXIES ASSOCIATED WITH INTERACTIONS}

Many of the studies summarized above were limited to pairs in which two distinct galaxies can be recognized, and where the morphological types of the components can be classified on a normal system. However there are several types of peculiar objects, such as ring galaxies, amorphous galaxies, or multiple-nucleus galaxies, which appear to form 
predominantly or exclusively from interactions. Since these systems represent some of the most extreme forms of interactions, it is useful to summarize their properties.

\section{Ring Galaxies}

Ring galaxies probably form as the result of the direct impact of an intruding mass through the center of a disk (Lynds and Toomre 1976, Theys and Spiegel 1976). Few and Madore (1986) examined the morphologies and environments of 69 southern ring systems, and found that those showing evidence of recent star formation also possess a higher than normal frequency of close companions. Appleton and Struck-Marcell (1987) used the IRAS database to determine $L_{F I R} / L_{B}$ ratios and $60 / 100 \mu \mathrm{m}$ color temperatures for 26 rings, and found that they exhibit the FIR excesses and high temperatures which are characteristic of active star forming galaxies. Detailed optical and near-infrared imaging of selected systems by Bonoli (1987) and Joy et al. (1988) show similar evidence of recent star formation.

\section{Amorphous (I0) Galaxies}

Hubble and Lundmark both recognized the existence of a subpopulation of irregular galaxies, typified by M82, which could not be placed in the normal morphological sequence, and galaxies of this type are referred to variously as Irregular II, I0, or Amorphous galaxies. While it is likely that this collection of outcasts from the Hubble sequence may have several formation mechanisms (Krienke and Hodge 1974), the presence of tidal bridges and tails around several systems has led to the suggestion that most may be formed in interactions or mergers (e.g., Cottrell 1978). Keel et al. (1985) noted that the frequency of amorphous galaxies in a complete sample of close pairs is 5 times higher than in the field, lending additional support to this idea. Not all such galaxies can be readily identified with interactions, however. Gallagher and Hunter (1987) found evidence of recent interactions in only $20-60 \%$ of a sample of 16 nearby amorphous galaxies. This is very high fraction compared to what would be expected if amorphous galaxies were a normal population, but it does suggest that other formation mechanisms may be necessary.

Several recent observations indicate that amorphous galaxies are often the sites of large global star formation bursts. Gallagher and Hunter (1987) found that the mean SFRs in their sample, as derived from both UBV colors and $\mathrm{H} \alpha$ fluxes, are essentially the same as in a comparison sample of active star forming Magellanic irregulars. The detection of compact radio sources, probably supernova remnants, in M82 and NGC 3448 by Kronberg et al. (1985) and Noreau and Kronberg (1987) adds support to this conclusion. In many of these galaxies the intense star formation activity is confined to the near-nuclear regions (Gallagher and Hunter 1987), a result which is reminiscent of Bushouse's (1987) result for interacting spirals.

\section{Mergers and Multiple Nucleus Galaxies}

The identification of the most luminous IRAS galaxies with mergers, combined with the suggestion that elliptical galaxies may form via mergers (e.g., Toomre 1977), has led to a widespread interest in these objects. As discussed earlier the nature of the enormous infrared luminosities of the IRAS galaxies is very controversial, but there is mounting evidence for global star formation bursts in many of these objects. The most direct evidence comes from near-infrared imaging (e.g., Stanford and Balcells 1990), which can penetrate the dust with shrouds the cores of many mergers, but unfortunately such data are only available for a few objects. The observation of strong, spatially extended Balmer absorption lines in the composite spectra of several mergers provides indirect evidence for enormous fossil $\left(\tau \sim 10^{8} y r\right)$ starbursts (Schweizer 1978, 1990; Hamilton and Keel 1987).

It has long been recognized that multiple-nucleus galaxies are unusually common in the Markarian sample (Petrosian, Saakyan, and Khachikian 1978). Systematic studies of the structure and spectroscopic properties of these galaxies have been conducted by Petrosian et al. (1980a, b) and Mazzarella (1989): The mere existence of these galaxies in such large 
numbers suggests a causal connection between close encounters/mergers and nuclear and/or disk activity. Perhaps the most notable property of the class is an unusually high fraction of Seyfert nuclei (Mazzarella 1989), though this may partly be a selection effect in the Markarian survey itself.

Schweizer (1990) summarizes a long series of observations in support of the hypothesis that mergers were the primary formation mechanism for elliptical galaxies and spiral bulges. The evidence, in addition to the observations summarized above, includes the $r^{1 / 4}$ luminosity distributions in many mergers, and the presence of counter-rotating cores in both mergers and some E/S0 galaxies (discussed extensively elsewhere in this volume). Although the role of mergers in galaxy formation is still unclear, especially from an observational perspective, I do believe that the data point to a very different mode of starburst activity in these objects, relative to what is seen in normal disks. The presence of enormous $\left(L \sim 10^{11-13} L_{\odot}\right)$ infrared luminosities, dense and massive central molecular disks, large amounts of interstellar dust, spheroidal morphologies, and Balmer-dominated spectra all represent more than a simple extrapolation of the star formation properties on normal disks; these are phenomena which appear to be almost uniquely identified with mergers. It is very tempting to associate these activities with the formation of stars in galactic spheroids and/or nuclei, regardless of their association with proto-elliptical galaxies and bulges.

\section{DEPENDENCE OF ACTIVITY ON INTERACTION PARAMETERS}

Considerable effort has been directed at understanding the parameters and processes which regulate the starbursts in interacting systems. The traditional approach to this question has been to correlate the emission levels with various interaction parameters, such as pair separation or velocity difference. Another approach is to start with what we know (or think we know!) about the star formation properties of normal galaxies, and examine these same trends in the interacting systems. The latter approach provides some interesting physical perspectives, so I begin there.

The SFR in isolated galaxies is most strongly correlated with Hubble type and gas content, as illustrated in Figures 2 and 3, respectively. In each case the SFR tracer plotted is the integrated equivalent width of the $\mathrm{H} \alpha$ emission line, which provides a measure of the total SFR in the galaxies, normalized to total (continuum) luminosity. Among isolated galaxies (labelled the "control sample" in each plot), there are strong correlations between this SFR and both galaxy type and gas density, albeit with considerable scatter about the mean.

When the same comparisons are made for interacting galaxies, however, these correlations largely disappear. The SFR in an interacting galaxy is virtually independent of its morphological type or gas content, and hence is effectively decoupled from its star formation properties prior to the encounter. Apparently interactions do not merely amplify a pre-existing level of star formation, they induce bursts which are regulated by mechanisms which are quite distinct from those which control the steady-state star formation in disks.

\section{The Holmberc Effect}

One of the best demonstrations of the dominating influence interactions can have on the global SFR is the "Holmberg effect," the tendency for the individual members of a pair to exhibit similar integrated colors (Holmberg 1958, Ardeberg and Bergvall 1977, Metik and Pronik 1978, Demin et al. 1984, Madore 1986). Part of the correlation is due to morphological segregation, the tendency for pairs to contain galaxies of similar morphological type (see the paper by Yamagata in this volume), but in many pairs the dominant cause is simultaneous star formation bursts in the two galaxies (Demin et al. 1984. Madore 1986). This is particularly true of the bluest pairs $(B-V<0.5)$, because such blue colors can only be produced in short-lived star formation bursts (e.g., Searle et al. 1973). 
Similar correlations are also seen in the $\mathrm{H} \alpha$ equivalent widths (Kennicutt et al. 1987) and FIR properties (Cutri, private communication) of close pairs.
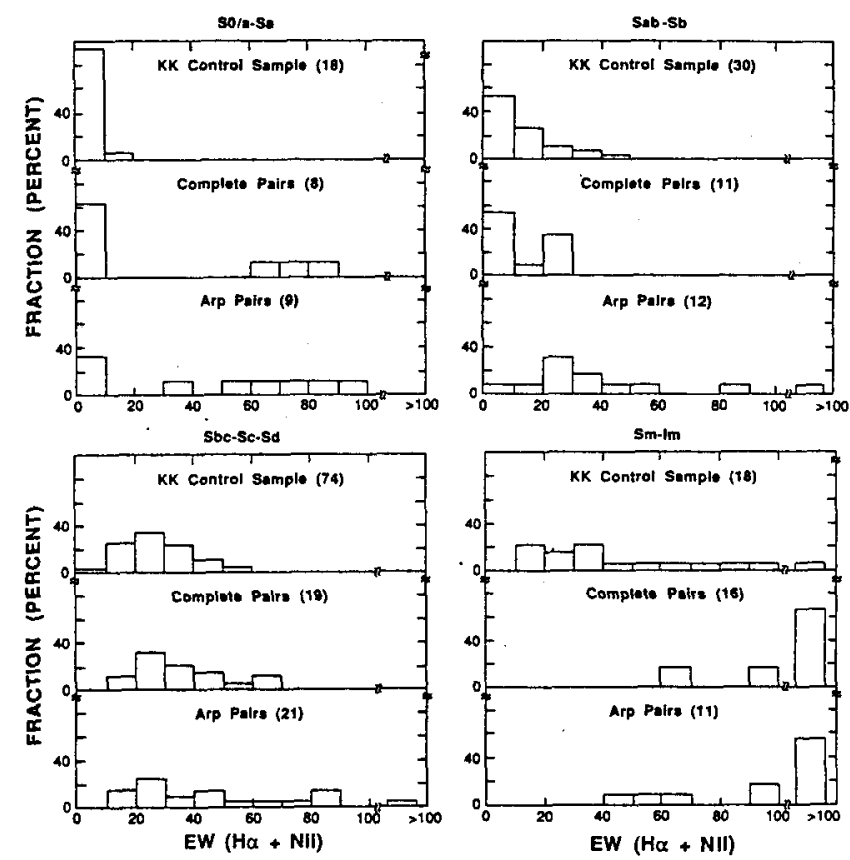

Fig. 2. Distributions of $\mathrm{H} \alpha$ emission line strengths for isolated (control) and interacting (complete, Arp) galaxies, subdivided by Hubble type.

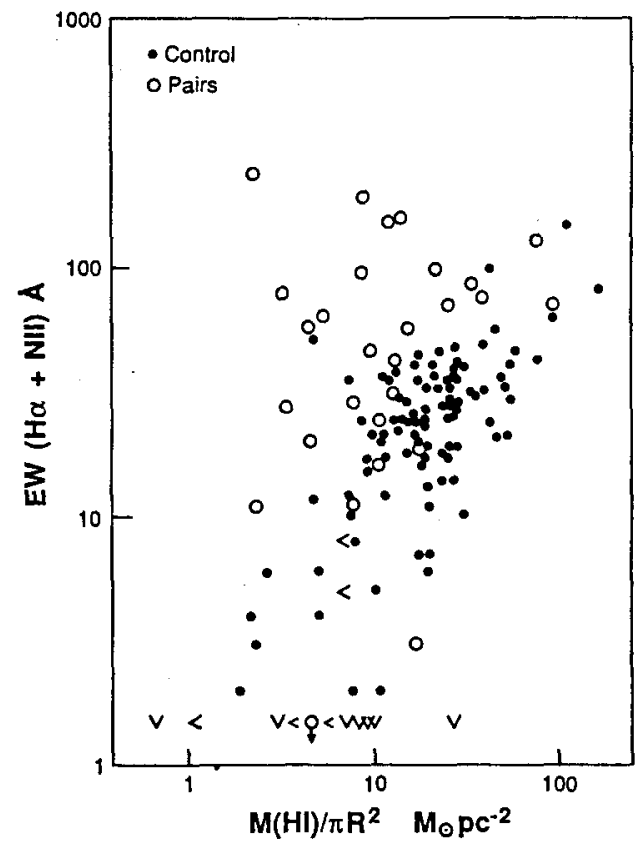

Fig. 3. Dependence of $\mathrm{H} \alpha$ emission on the surface density of atomic hydrogen, for isolated (solid points) and interacting galaxies (open circles).

\section{Dependence on Pair Separation and Velocity Difference}

The tidal perturbations in an interaction are the strongest for close, slow encounters, so if dynamical parameters regulate the SFR one would expect the emission levels to be inversely correlated with the projected separations and velocity differences of the pairs. Both trends are observed. Closer pairs show an excess of Markarian galaxies (Casini and 
Heidmann 1975, Karachentsev 1981), bluer colors (Madore 1986), higher average levels of $\mathrm{H} \alpha$ emission (Tifft 1985, Kennicutt et al. 1987) stronger FIR emission (e.g., Bushouse et al. 1988, Jones and Stein 1989), and hotter FIR color temperatures (Telesco et al. 1988).

Figure 4, taken from Jones and Stein (1989), illustrates the dependence of the FIR/B ratio on separation, and provides a case study in some of the ambiguities inherent in this sort of analysis. The unmarked points are pairs taken from the Bushouse et al. (1988) opticallyselected sample, while the labeled points are ultraluminous IRAS galaxies taken from Sanders et al. (1988). The mean level of FIR emission shows a weak separation dependence at best, but the upper envelope of the distribution rises rapidly with decreasing separation, especially when the ultraluminous galaxies are included. This is an excellent illustration of the nearly unique association of the ultraluminous FIR galaxies with close violent mergers, but these objects are so rare and so distinct in their properties that their separation distribution should probably be considered separately from that of the Bushouse sample.

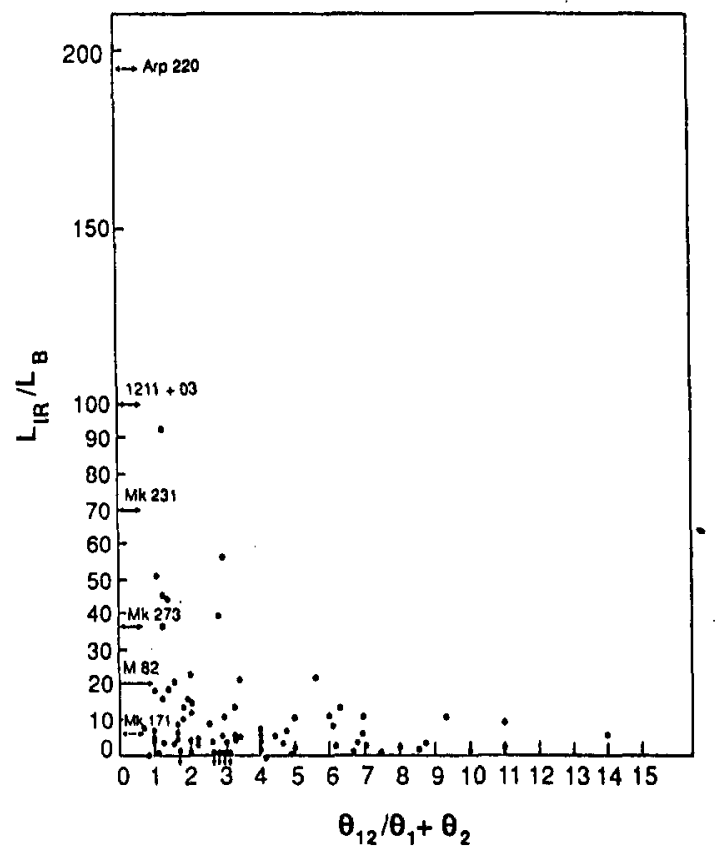

Fig. 4. Dependence of FIR emission on pair separation for a sample of interacting galaxies studied by Jones and Stein. Ultraluminous FIR galaxies are labeled individually.

Less is known about the dependence of emission properties on the radial velocity separations of the components. Karachentsev (1981) demonstated that pairs containing Markarian galaxies show significantly smaller differences in velocity than normal pairs. Tifft (1985) examined the redshift differences in a sample of 250 close pairs, and found a pronounced increase in the frequency of emission line detections with decreasing velocity difference. Tifft also investigated the dependence of emission properties on the product of velocity separation and projected linear separation, and finds a correlation there as well. Unfortunately his (photographic) spectra are uncalibrated, so a more quantitative analysis would be very useful.

While these sorts of statistical studies provide convincing qualitative confirmation of the expected dependences of the SFRs on orbital properties, the trends are so noisy that a detailed physical interpretation is not yet possible. The large dispersion in emission properties is not unexpected if one considers the wide range in projection angles, orbital velocities, orientations of rotation and orbital axes, and evolutionary stages. While these problems may be redressed somewhat by observing larger samples, the most productive observations in the future may be those which match detailed kinematic observations of individual pairs with dynamical simulations (e.g., Jenkins 1984, Borne 1988a, b; Borne and Hoessel 1988, Hernquist 1990). 


\section{INTERPRETATION AND MODELLING}

In contrast to the extensive observational work described above, relatively little effort has been devoted to the modelling of these data in order to to derive the evolutionary properties of the systems. Fortunately the original Larson and Tinsley (1978) model has stood up very well against the new observations, and it provides an excellent starting point for this discussion.

\section{Properties of Star Formation Bursts}

Figure 5, taken from the LT paper, shows the observed $U B V$ colors of normal (left) and peculiar galaxies from the Arp atlas (right), together with curves which depict the expected colors of model stellar populations. The normal galaxies are well fitted by a sequence of fixed-IMF, fixed-age models with variable star formation histories (cf. Searle et al. 1973). On the other hand the blue colors of many of the interacting galaxies require strong star formation bursts superimposed on the underlying populations. The upper envelope to the observed color distribution requires relatively short-lived bursts, $20 \mathrm{Myr}$ in the case of the upper curve in Fig 5b. The lower envelope of the color distribution imposes a limit on the strength of the burst (i.e., the fraction of the total mass of stars in the galaxy formed in the bursts), and for the Arp sample the inferred burst strengths are all below 5\%. Hence the blue colors of the interacting galaxies can be reproduced with very modest starbursts, in terms of the total mass of stars formed, but the models do require that the star formation take place over a time interval which is short compared to the dynamical time scales of most encounters.

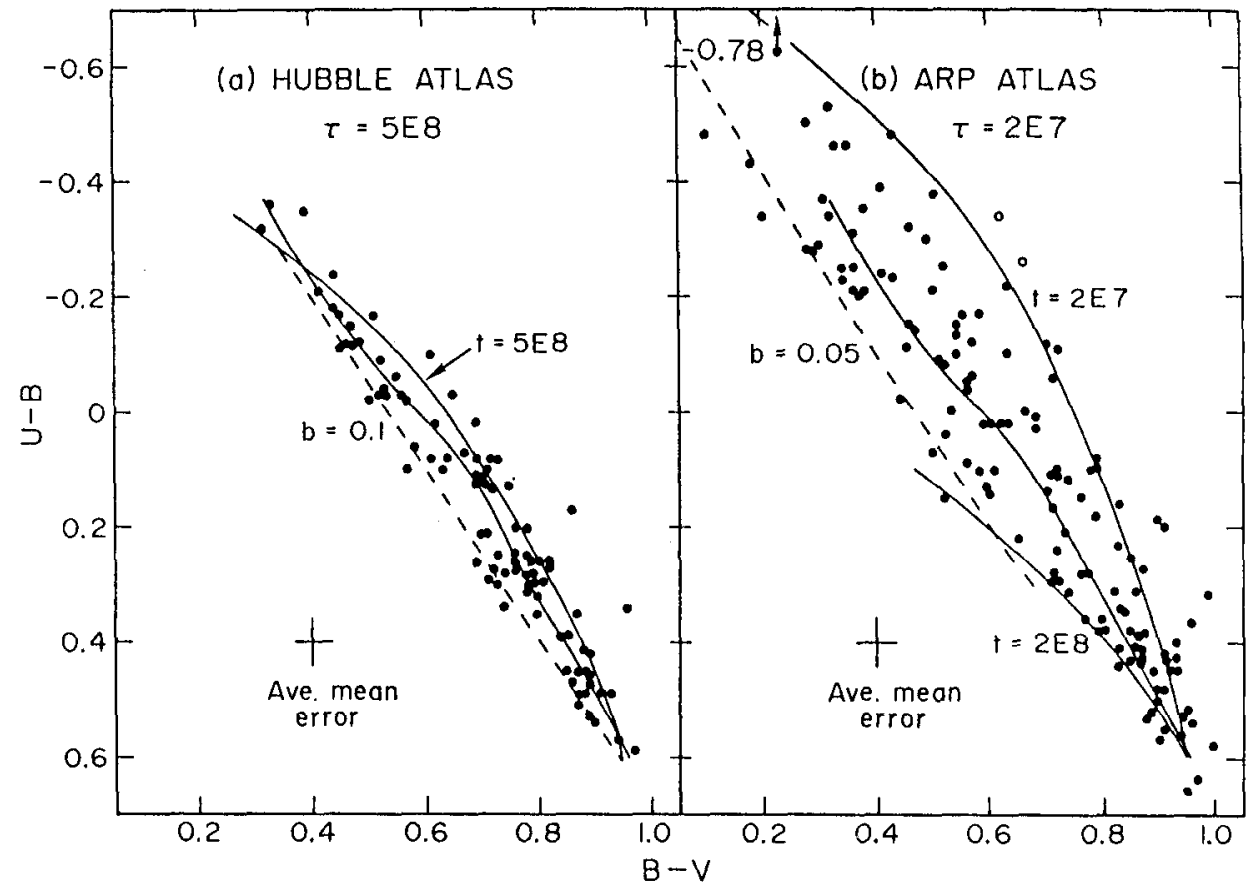

Fig. 5. Distribution of UBV colors of normal and peculiar (mostly interacting) galaxies, along with evolutionary models fitted to the data, from Larson and Tinsley (1978). See text for a description of the models.

Kennicutt et al. (1987) extended this approach by calculating the evolution of the $\mathrm{H} \alpha$ emission line fluxes and equivalent widths as well as the colors. The $\mathrm{H} \alpha$ fluxes provide a direct measurement of the SFR during the interaction, as well as independent constraints on the time scales and initial mass function (IMF) of the bursts. We found that the $\mathrm{H} \alpha$ equivalent widths and colors for most interacting galaxies could be fitted with burst 
properties falling within the LT parameter space, namely durations of less than $20 \mathrm{Myr}$, a normal (Salpeter) IMF, and a total mass in new stars of a few percent or less of the total disk mass. Over the entire sample, the colors can be fitted with bursts of duration 3-50 Myr, amplitudes of order 0.1-100 times the pre-existing SFR, and absolute SFRs of order 0.1-100 $\mathrm{M}_{\odot} \mathrm{yr}^{-1}$. These imply total masses of up to $10^{9}-10^{10} \mathrm{M}_{\odot}$ in stars formed over the duration of the burst.

An independent upper limit on the lifetime of the star formation bursts can be imposed by comparing the peak SFR to the total mass of interstellar gas available in the star forming region. In strong starburst galaxies these yield total consumption times of order 0.1-1 Gyr if the total gas mass is used (i.e., $100 \%$ star formation efficiency), and proportionally less if a smaller total efficiency is assumed. The latter are consistent with the photometrically inferred time scales.

These models barely manage to reproduce the very high $\mathrm{H} \alpha$ fluxes and equivalent widths of the most active starburst galaxies, those lying in the upper envelope of Fig. 5 . In the standard LT model these extreme photometric properties require very young $(\tau \ll 10 M y r)$ starbursts, but such short-lived bursts appear to be inconsistent with the fraction of interacting galaxies observed in this stage. The only alternative is to question the assumptions underlying the models, and of those the most likely culprit is the assumed IMF.

\section{Initial Mass Function and Star Formation Efficiency}

Although most models for the evolution of interacting galaxies presume for simplicity's sake a universal IMF, there is accumulating evidence for abnormal IMFs in the most active star forming systems. Unfortunately, constraining even the rough form of the IMF in these galaxies is itself a highly model-dependent exercise, and in some cases the various determinations are mutually inconsistent. For example, the blue colors and high Balmer emission-line equivalent widths in the most active galaxies are best understood if the IMF is heavily enriched in massive stars $\left(M=10-100 \mathrm{M}_{\odot}\right)$ relative to the solar neighborhood (Huchra 1977, Kennicutt et al. 1987). On the other hand, the high fraction of nonthermal radio emission in these objects (as compared to ordinary giant H II regions) has led Gehrz, Sramek and Weedman (1983) and Nakagawa et al. (1989) to suggest a deficiency of stars more massive than $30 \mathrm{M}_{\odot}$.

The most detailed model of the IMF in a starburst region has been constructed for M82 by Rieke et al. (1980). In addition to using the ionizing properties to constrain the upper IMF, they used the dynamical mass and IR luminosity of the region to investigate the IMF at low stellar masses. Their best fitting models require both upper and lower mass cutoffs to the IMF, with a typical range of 5-30 $\mathrm{M}_{\odot}$. Although more recent models by Bernlohr (this conference) relax the lower mass limits considerably, the observed properties of the starbursts are still difficult to reconcile with a normal IMF.

Careful examination of these various models reveals a complex interplay between the IMF parameters and the temporal properties of the starbursts, and I suspect that the constraints on the IMF are considerably weaker than the authors (this one included) would hope. Another fundamental issue which has yet to be addressed conclusively is whether the unusual IMFs attributed to these regions are unique to interacting (or luminous starburst) galaxies, or whether they merely reflect a general difference or bimodality in the IMF between active star forming regions and the integrated population of an entire galaxy.

Some of these models also suggest unusually high efficiencies of star formation in the starbursts. In the M82 core, for example, the observed luminosities and gas contents require efficiencies of order 10-100\%, regardless of the IMF (Rieke et al. 1980). This dilemma can be relaxed if much of the gas involved in the star formation has been driven away, but it is difficult to see how this could explain the entire difference in efficiencies. Again it is important to note that this problem may not be unique to interacting or IR-luminous galaxies (cf. Kennicutt and Chu 1988). The unusually high ratios of FIR to CO molecular line luminosity in many interacting galaxies has been used to argue for abnormally high global star formation efficiencies (e.g., Young et al. 1986, Solomon and Sage 1988). This 
result verifies that the SFRs in these galaxies are not the result of abnormally high gas contents.

Global Role of Interactions in Star Formation and Galactic Evolution

An interesting quantity which can be derived from the evolution models is the fraction of star formation in the universe which is triggered by interactions. Kennicutt et al. (1987) combined their SFR study of a complete sample of pairs with the fraction of such pairs in the Shapley-Ames catalog, and estimated that interactions are responsible for $6 \pm 3 \%$ of the current star formation in nearby spiral galaxies. The main uncertainties in this result are the definition of an interacting galaxy, Malmquist bias in the pairs catalog, and the assumption that the IMF in the interacting systems is the same as in normal spirals.

This fraction was probably much higher at earlier cosmological epochs, and it is becoming possible to test this hypothesis observationally. Lavery and Henry (1988) show that most of the blue galaxies in a rich cluster at $z=0.2$ possess companions or other visible evidence of recent interactions, and they propose that interactions may be the primary cause of the Butcher-Oemler effect in more distant clusters. Along similar lines Zepf and Koo (1989) have used a deep survey of distant field galaxies to compare the frequency of close pairs at faint magnitudes $(B \leq 22)$ with the fraction among bright, nearby galaxies. They find a statistically significant excess of faint pairs, which is consistent with an increase in the frequency of interactions $f \propto(1+z)^{4.0 \pm 2.5}$. This implicitly assumes that the detection of the faint pairs has not been biased by luminosity evolution effects, so the conclusion remains tentative until better data can be obtained.

As an amusing, albeit speculative exercise, one can combine the Kennicutt et al. fraction of current star formation from interactions with the Zepf and Koo redshift dependence for the interaction frequency, in order to estimate the redshift at which interactions dominate the global star formation in galaxies. Using the numbers above, including all of the uncertainties, yields a range of 'formation' redshifts $z=0.5-3$. Systematic errors tend to bias this result in the direction of lower $z$, but this back of the envelope calculation does emphasize the likely importance of interactions at early epochs, even if their global role is quite minor at the current time.

\section{CONCLUDING REMARKS}

The main accomplishment in this field over the past decade has been the quantitative verification, sometimes in spectacular fashion, of the Larson and Tinsley (1978) hypothesis that interactions are an important trigger of star formation bursts in galaxies. The secondorder questions, such as the dependence of the star formation on the physical and kinematic properties of the pairs, the relative roles of nuclear and extranuclear emission, etc., are less well understood, but considerable progress in these areas can be expected over the next few years.

Now that the easy work has been done, further interpretation of the statistics of optical, IR, and radio emission properties of these galaxies is becoming increasingly limited by fundamental uncertainties in our physical understanding of the nature of the stellar populations and the dynamical properties of the interactions themselves. Among the most important questions:

1. What is the physical nature of the "starbursts" in interacting galaxies, and how many starburst phenomena are we observing? Most current surveys implicitly assume that we observing a single generic type of star formation activity, but the recent observations, particularly those at infrared wavelengths, suggest the presence of at least two distinct modes of activity, with very different dependences on interaction properties. The simplest consideration of galactic structure and evolution suggests that we should see at least three distinct types of starbursts, one associated with an enhanced level of disk star formation, another associated with the formation of galactic spheroids, and perhaps another associated 
with the formation of the dense mass concentrations in the centers of galaxies. Before we can fully understand the nature of the "starburst phenomenon" we need to establish how many processes we are actually observing.

2. What are the physical mechanisms which trigger and regulate the starbursts? Both observations and theory suggest that gas dynamics, possibly coupled to a strong star formation threshold (e.g., Quirk 1972, Kennicutt 1989) are central elements of the triggering mechanism, but to date relatively little observational effort has been devoted toward measuring the dynamical properties of the stars and gas in interacting galaxies. The recent discoveries of $\mathrm{U}$-shaped rotation curves and counter-rotating cores in isolated $\mathrm{E}$ galaxies and mergers are impressive illustrations of how important such observations can be.

3. What is the nature of the star formation in the starbursts, especially the IMF, relative to that in normal star forming galaxies? This question is of more than pure theoretical interest. The interpretation of virtually all photometric tracers of star formation rates in galaxies, regardless of wavelength, rests heavily on the assumed form of the IMF. Until the IMF is understood both our observational and theoretical understanding of the evolution of these objects will be severely handicapped.

Significant future progress in our understanding of the evolution of interacting (and normal!) galaxies will require more than a mere extention of current surveys to larger samples. Observations which will be especially crucial for disentangling the questions cited above include detailed kinematic studies of individual systems, preferably coordinated with simulations of the dynamics of the encounters, high-resolution maps of the atomic and molecular gas distributions and kinematics, and multi-wavelength observations of the stellar populations in a range of systems. Such observations may lead not only to a better understanding of tidal interactions, but also to important insights into the mechanisms regulating galactic evolution in general.

I am grateful to my collaborators W. Keel, E. Hummel, and J. M. van der Hulst, as well as H. Bushouse, R. Cutri, T. Heckman, A. Petrosian, S. White, and A. Zasov for very useful discussions about this subject. I also thank H. Bushouse, R. Larson, and W. Stein for permission to reproduce their figures in this paper. This work was supported in part by NSF Grant AST89-96123.

\section{REFERENCES}

Appleton, P. N., and Struck-Marcell, C. 1987, Ap. J., 312, 566.

Ardeberg, A., and Bergvall, N. 1977, Astr. Ap., 61, 493.

Arkhipova, V. P. 1982, Astr. Zh., 59, 209.

Armus, L., Heckman, T., and Miley, G. 1987, A. J., 94, 831.

Bergvall, N. A. S. et al. 1978, Astr. Ap. Suppl., 33, 243.

Bonoli, C. 1987, Astr. Ap., 174, 57.

Borne, K. D. 1988a, Ap. J., 330, 38.

Borne, K. D. $1988 \mathrm{~b}, A p$. J., 330, 61.

Borne, K. D., and Hoessel, J. G. 1988, Ap. J., 330, 51.

Bothun, G. D., Halpern, J. P., Lonsdale, C. J., Impey, C., and Schmitz, M. 1989, Ap. J. Suppl., 70, 271.

Bushouse, H. A. 1987, Ap. J., 320, 49.

Bushouse, H. A., Lamb, S. A., and Werner, M. W. 1988, Ap. J., 335, 74.

Casini, C., and Heidmann, J. 1975, Astr. Ap., 39, 127.

Condon, J. J. 1983, Ap. J. Suppl., 53, 459.

Condon, J. J., Condon, M. A., Gisler, G., and Puschell, J. J. 1982, Ap. J., 252, 102.

Cottrell, G. A. 1978, M.N.R.A.S., 184, 259. 
Cutri, R. M., and McAlary, C. W. 1985, Ap. J., 296, 90.

Demin, V. V., Dibai, E. A., and Tomov, A. N. 1981, Astr. Zh., 58, 925.

Demin, V. V., Zasov, A. V., Dibai, E. A., and Tomov, A. N. 1984, Astr. Zh., 61, 625.

Dostal', V. A. 1982, Armenian Astrofisica, 18, 201.

Few, J. M. A., and Madore, B. F. 1986, M.N.R.A.S., 222, 673.

Fried, J. W., and Lutz, D. 1988, Astr. Ap., 197, 52.

Friedman, S. D., Cohen, R. D., Jones, B., Smith, H. E., and Stein, W. A. 1987, A. J., 94, 1480.

Gallagher, J. S., Hunter, D. A., and Tutukov, A. V. 1984, Ap. J., 284, 544.

Gallagher, J. S., and Hunter, D. A. 1987, A. J., 94, 43.

Gehrz, R. D., Sramek, R. A., and Weedman, D. W. 1983, Ap. J., 267, 551.

Hamilton, D., and Keel, W. C. 1987, Ap. J., 321, 211.

Haynes, M. P., and Herter, T. 1988, A. J., 96, 504.

Hernquist, L. 1990, in Dynamics and Interactions of Galaxies, ed. R. Wielen (Dordrecht: Kluwer), in press.

Hodge, P. W. 1975, Ap. J., 202, 619.

Holmberg, E. 1958, Medd. Lunds Astr. Obs., 2, No. 136.

Huchra, J. P. 1977, Ap. J., 217, 928.

Hummel, E. 1981, Astr. Ap., 96, 111.

Hummel, E., and van der Hulst, J. M. 1986, Astr. Ap., 155, 151.

Hummel, E., van der Hulst, J. M., Keel, W. C., and Kennicutt, R. C. 1987, Astr. Ap. Suppl., 70, 517 .

Hummel, E., van der Hulst, J. M., Kennicutt, R. C., and Keel, W. C. 1990, Astr. Ap., submitted.

Jenkins, C. R. 1984, Ap. J., 277, 501.

Johansson, L. 1988, Astr. Ap. Suppl., 73, 335.

Jones, B., and Stein, W. A. 1989, A. J., 98, 1557.

Joseph, R. D. 1987, in Starbursts and Galaxy Evolution, ed. T. X. Thuan, T. Montmerle, J. Tran Thanh Van (Paris: Editions Frontieres), p. 293.

Joseph, R. D. 1988, in Comets to Cosmology, ed. A. Lawrence (Berlin: Springer), p. 234.

Joseph, R. D., Meikle, W. P. S., Robertson, N. A., and Wright, G. S. 1984, M.N.R.A.S., 209, 111.

Joy, M., Ellis, H. B., Tollestrup, E. V., Brock, D., Higdon, J. L., and Harvey, P. M. 1988, $A p$. J. (Letters), 330, L29.

Joy, M., and Ghigo, F. D. 1988, Ap. J., 332, 179.

Karachentsev, I. D., and Karachentseva, V. E. 1974, Astr. Zh., 51, 724.

Karachentsev, I. D. 1981, Pis'ma Astr. Zh., 7, 3.

Keel, W. C., Kennicutt, R. C., Hummel, E., and van der Hulst, J. M. 1985, A. J., 90, 708.

Kennicutt, R. C. 1983, Ap. J., 272, 54.

Kennicutt, R. C. $1989, A p$. J., 344,685.

Kennicutt, R. C., and Chu, Y.-H. 1988, A. J., 95, 720.

Kennicutt, R. C., and Kent, S. M. 1983, A. J., 88, 1094.

Kennicutt, R. C., Keel, W. C., van der Hulst, J. M., Hummel, E., and Roettiger, K. A. 1987, A. J., 83, 1011.

Krienke, O. K., and Hodge, P. W. 1974, A. J., 79, 1242.

Kronberg, P. P., Biermann, P., and Schwab, F. R. 1985, Ap. J., 291, 693.

Larson, R. B., and Tinsley, B. M. 1978, Ap. J., $219,46$.

Lavery, R. J., and Henry, J. P. 1988, $A p$. J., 330, 596.

Lonsdale, C. J., and Helou, G. 1987, Ap. J., 314, 513.

Lonsdale, C. J., Persson, S. E., and Mathews, K. 1984, Ap. J., $287,95$.

Lynds, C. R., and Toomre, A. 1976, Ap. J., $209,382$.

Madore, B. F. 1986, in Spectral Evolution of Galaxies, ed. C. Chiosi and A. Renzini

(Dordrecht: Reidel), p. 97.

Mazzarella, J. 1989, Ph.D. thesis, University of Michigan. 
Metic, L. P., and Pronik, I. I. 1978, Astr. Zh., 55, 249.

Nakagawa, T., Nagata, T., Geballe, T. R., Okuda, H., Shibai, H., and Matsuhara, H. 1989, Ap. J., 340, 729.

Noreau, L., and Kronberg, P. P. 1987, A. J., 93, 1045.

Petrosian, A. R., Saakian, K. A., and Khachikian, E. E. 1978, Astrofisica, 14, 69.

Petrosian, A. R., Saakian, K. A., and Khachikian, E. E. 1980a, Pis'ma Astr. Zh., 6, 262.

Petrosian, A. R., Saakian, K. A., and Khachikian, E. E. 1980b, Pis'ma Astr. Zh., 6, 552.

Petrosian, A. R., Saakian, K. A., and Khachikian, E. E. 1985, Astrofisica, 21, 57.

Quirk, W. J. 1972, Ap. J. (Letters), 176, L9.

Rieke, G. H., Lebofsky, M. J., Thompson, R. I., Low, F. J., and Tokunaga, A. T. 1980, $A p$. $J ., \mathbf{2 3 8}, 24$.

Sanders, D. B., Soifer, B. T., Elias, J. H., Madore, B. F., Matthews, K., Neugebauer, G., and Scoville, N. Z. 1988, Ap. J., 325, 74 .

Scalo, J. M. 1986, Fund. Cos. Phys., 11, 1.

Schombert, J. M., Wallin, J. F., and Struck-Marcell, C. 1990, A. J., 99, 497.

Schweizer, F. 1978, in Structure and Properties of Nearby Galaxies, ed. E. M. Berkhuijsen and R. Wielebinski (Dordrecht: Reidel), p. 279.

Schweizer, F. 1990, in Dynamics and Interactions of Galaxies, ed. R. Wielen (Dordrecht: Kluwer), in press.

Searle, L., Sargent, W. L. W., and Bagnuolo, W. G. 1973, Ap. J., 179, 427.

Smirnov, M. A., and Tsvetkov, D. Yu. 1981, Pis'ma Astr. Zh., 7, 154.

Soifer, B. T., etal. 1984, Ap. J. (Letters), 278, L71.

Solomon, P. M., and Sage, L. J. 1988, Ap. J., 334, 613.

Stanford, S. A., and Balcells, M. 1990, A. J., in press.

Stocke, J. T. 1978, A. J., 83, 348.

Sulentic, J. W. 1976, Ap. J. Suppl., 32, 171.

Sulentic, J. W. 1989 A. J., 98, 2066.

Telesco, C. M. 1988, Ann. Rev. Astr. Ap., 26, 343.

Telesco, C. M., Decher, R., and Gatley, I. 1985, Ap. J., 299, 896.

Telesco, C. M., Wolstencroft, R. D., and Done, C. 1988, Ap. J., 329, 174.

Theys, J. C., and Spiegel, E. A. 1977, Ap. J., 212, 616.

Tifft, W. G. 1985, Ap. J., 288, 65.

Tinsley, B. M., and Danly, L. 1980, Ap. J., 242, 435.

Toomre, A. 1977, in The Evolution of Galaxies and Stellar Populations, ed. B. M. Tinsley and R. B. Larson (New Haven: Yale Univ.), p. 401.

Toomre, A., and Toomre, J. 1972, Ap. J., 178, 623.

Walterbos, R. A. M., and Schwering, P. B. W. 1987, Astr. Ap., 180, 27.

Wasilewski, A. J. 1983, Ap. J., 272, 68.

Young, J. S., Schloerb, F. P., Kenney, J. D., and Lord, S. D. 1986, Ap. J., 304, 443.

Zepf, S. E., and Koo, D. C. 1989, Ap. J., 337, 34. 


\section{DISCUSSION}

\section{Bland:}

For NGC 4647, where an extended population of HII regions cut off suddenly and the $\mathrm{HI}$ is observed to extend much further, if there is no evidence for bars or external dynamics, you probably have direct evidence of the importance of disk self-gravity. Have you measured the gas stability in this object, say, with Toomre's criterion?

Rennicutt: Yes! In a recent study of the star formation law in disks (Kennicutt 1989), I compared to locations of the observed star formation thresholds with the predicted gravitational stability threshold according to the Toomre criterion. There is a remarkable agreement between the predicted and observed thresholds, which suggests that gravitational stability may be the main regulator of the large scale star formation in late-type galaxies (including NGC 4647). This confirms a long-standing prediction by Quirk (1972).

Burbidge: Surely your last point is the most important one. Until you can place limits on the behavior of the IMF in these systems, all of the conclusions concerning star formation and evolution are meaningless.

Kennicutt: The situation is not quite that bad! The agreement in the star formation rates derived from $\mathrm{H} \alpha$, UV, and UBV data, all which trace different parts of the IMF, tells us that the rates cannot be in error by more than factors of a few, and hence the orders-of-magnitude bursts which are observed in many interacting systems cannot be simply artifacts of an unusual IMF. However, I wholly agree that our ignorance about the IMF in these galaxies is the main impediment to understanding their retailed evolutionary properties. 